\title{
Polymer Extraction from Processed Lignocellulosic Biomass Water Hyacinth (Eichhornia crassipes) for the Potential Biological Activities
}

\author{
Avinash G.P. ${ }^{1(\mathbb{D})}$, Karthick Raja Namasivayam S. ${ }^{2, * \mathbb{C}}$, Bavani Latha M. ${ }^{3(\mathbb{D}}$, Arvind Bharani R.S. ${ }^{4(\mathbb{D})}$ \\ 1 Centre for Bioresource research\& Development (C-BIRD),Department of Biotechnology, Sathyabama Institute of Science \\ \&Technology, Chennai 119, Tamil Nadu, India; govindarajavinash@gmail.com (A.G.P.); \\ 2 Centre for Bioresource research \& Development (C-BIRD), Department of Biotechnology, Sathyabama Institute of \\ Science \&Technology, Chennai 119, Tamil Nadu, India; biologiask@ gmail.com (K.R.N.); \\ 3 Department of Biotechnology, Sathyabama Institute of Science \&Technology, Chennai 119, Tamil Nadu, India; \\ bavagold@gmail.com (B.L.M.); \\ 4 Centre for Bioresource Research \& Development (C-BIRD), Department of Biotechnology, Sathyabama Institute of \\ Science \&Technology, Chennai 119, Tamil Nadu, India; arvindbharani@gmail.com (A.B.R.S.); \\ * Correspondence: biologiask@gmail.com;
}

Received: 26.07.2020; Revised: 4.09.2020; Accepted: 6.09.2020; Published: 10.09.2020

\begin{abstract}
Eichhornia crassipes (Water hyacinth) is considered a hazardous weed in numerous places in the world. Notwithstanding its natural disintegrating impacts, the weed, despite everything, offers the potential to be utilized as a substrate for the production of industrially important products. In this study, biological activities of water hyacinth biomass-derived cellulose composite were prepared and checked for biological activities. Cellulose was extracted from acid-alkali treated biomass of water hyacinth followed by fabrication with chlorpyrifos pesticide and curcumin. Potential biological activities of the prepared composite were also studied. The effect of solvents like ethanol, phosphatebuffered saline, and distilled water on the release of pesticide and curcumin was studied under in vitro condition. Powdered biomass treated with acid-alkali brought about highly stable, crystalline, cellulose with a final yield of $22.4 \mathrm{~g} / 100 \mathrm{~g}$ of dry weight. Characterizationtechniquesreveals structural modification of extracted cellulose with chlorpyrifos, curcumin, and the conversion of fabricated material as composite. Among the solvents tested, ethanol recorded maximum release of chlorpyrifos, curcumin at all the tested time periods, and the biological activities were also found to be increased in ethanol released samples. Structurally, functionally stable cellulose extracted from water hyacinth biomass and its derived composite material would suggest noteworthy bioresource utilization approaches of versatile invasive aquatic weeds.
\end{abstract}

Keywords: Eichhornia crassipes; biomass; composite; pesticide; curcumin; release.

(C) 2020 by the authors. This article is an open-access article distributed under the terms and conditions of the Creative Commons Attribution (CC BY) license (https://creativecommons.org/licenses/by/4.0/).

\section{Introduction}

Lignocellulosic biomass has been progressively examined as of late, in light of the fact that its utilization as a vitality source doesn't straightforwardly rival nourishment creation. Moreover, the change of lignocellulosic squander by methods for warm or biochemical procedures can deliver fluid powers, for example, ethanol or bio-oil, vaporous energize, for example, biogas (methane), and power [1]. Other positive perspectives to utilizing lignocellulosic biomass as feedstock remember overall conveyance and less impact on global warming [2,3]. Lignocellulosic feedstock speaks to an uncommonly enormous measure of 
inexhaustible bioresource accessible in surplus on earth and is an appropriate crude material for a huge number of utilizations for human manageability [4].

Among the different lignocellulosic biomasses, aquatic biomass has gotten more consideration in manageable vitality age and value-added industrial important products [5]. Aquatic biomass is made out of different types of miniaturized scale and macroalgae and aquatic plants [6]. Aquatic weeds have astoundingly higher propagation rates and are wealthy in cellulose and hemicellulose with a low lignin content that makes them an effective cuttingedge biofuel crop [7].

Aquatic weeds are the plants that grow for the most part or totally in water, whether set up in the mud, like a lotus or drifting without port, as the water hyacinth. Oceanic weeds give a critical peril to the freshwater stores (lake, stream, lake) and are the explanation behind Environmental sullying [8,9]. All lakes, from minimal shallow lakes, to greater lakes, can get smothered with weeds, especially where there is supplement rich run-off from enveloping cultivating territory. These structure thick, ugly tangles over the whole water surface. At the overall level, the yearly mishaps in view of the proximity of harming weeds are resolved to connect with $10-15 \%$ of creation among staple yields. There are starting at now 227 weed species recognized to be responsible for $90 \%$ of mischief to harvests, 18 of which are the most ruinous around the globe. Along these lines, it isn't exceptional that $47.5 \%$ of the 2 million tons of pesticides ate up comprehensive consistently are herbicides [10].

Among the various aquatic weeds, water hyacinth (Eichhornia crassipes) is a pleustophytichydatophyte, a cosmopolitan maritime weed; it is commonly seen as the world's most discernibly horrendous maritime weed. This weed structures thick immune tangles across the water surface, obliging access by man, animals, and device. Furthermore, course and calculating are obstructed, and water framework similarly as waste systems become blocked. This plant remains handy for quite a while past what six years, and new plants can be recuperated from seeds and parts of stem license the fast augmentation of plants [11].

Water Hyacinth outlines colossal tangles on still and slow-moving water bodies, where it genuinely corrupts maritime organic frameworks and limits their utilization. It has been recorded as one of the most gainful plants on earth, which has assaulted freshwater structure in excess of 50 countries on five territories, especially all through Southeast Asia, the southeastern United States, Central and western Africa. They can persevere through a wide extent of environmental conditions, for instance, temperature, lighting up, $\mathrm{pH}$, saltiness, wind, rhythmic movement, and drought. The plant is morphologically extraordinarily plastic with a quick strategy for the vegetative spread, which makes it especially changed in accordance with long division dispersal and productive colonization of grouped organic claims to fame. It is one of the most beneficial maritime plants which spread at an upsetting rate having spikes of gigantic blue blooms and roundish leaves with expanding bladder like petioles [12].

Ignoring its regular debilitating effects, the weed, in spite of everything, offers potential to be used as a substrate for the production of monetarily critical things because of its promising sugar content, several regards included things can be conveyed from WH development. This fuse different synthetic compounds, cellulose xanthogenate, levulinic destructive, shikimic destructive, biogas, bioethanol, biohydrogen, biopolymer, biobutanol, composites, biofertilizers, fish feed, high calorific worth fuel, fuel briquette, superabsorbent polymer, and xylitol [13]. Zhang et al. [14] revealed the noteworthy utilization of various aquatic weeds into important commercial products like ethanol and bio-oil. Industrial significant biopolymer like poly hydroxyl alkanoates production using acid-treated WHB with the aging standards of 
bacterial strain Pseudomonas aeruginosa has contemplated by Preethi et al. [15]. Heavy metals adsorption efficacy of metal accumulating property and environmental pollutants removal efficacy of water hyacinth biomass have been reported [16,17]. In this present study, cellulose was extracted from acid-alkali treated biomass of water hyacinth biomass (WHB), and the extracted cellulose was fabricated with chlorpyrifos pesticide, curcumin, transformed into stable composite material with potential biological activities.

\section{Materials and Methods}

\subsection{Sample collection and processing.}

Water hyacinth was collected from the ponds in Neelangarai, ECR, and kept in the laboratory for further purposes. The collected biomass was washed with tap water, followed by distilled water. The washed material was dried in a hot air oven at $50^{\circ} \mathrm{C}$ for $12 \mathrm{hrs}$. After drying, the biomass was ground with a domestic mixer, and the powder was sieved and collected in a separate container.

\subsection{Extraction of cellulose.}

Acid- alkali treatment was adopted for the extraction of cellulose. A known quantity of processed biomass $(30 \mathrm{~g})$ was treated with $1.0 \mathrm{M} \mathrm{HNO}_{3}$ for 24 hours under a stirrer. After stirring, the acid-treated biomass was filtered through filter paper, and the collected solid material was transferred to the conical flask, followed by the dropwise addition of alkali solution $(1.0 \mathrm{M} \mathrm{NaOH})$ under stirring for another 24 hours. Alkali treated content was filtered through filter paper, residues thus obtained was washed with sterile distilled water, and the washed cellulose material was dried at $50^{\circ} \mathrm{C}$, used for further studies.

\subsection{Preparation of cellulose grafted Chlorpyrifos pesticide composite.}

A simple dispersion method was adopted for the preparation of cellulose grafted chlorpyrifos composite. A commercial formulation of chlorpyrifos(obtained from an agriculture department store) was used in this study. Extracted cellulose $(100 \mathrm{mg})$ dispersed in 1.0 $\mathrm{M} \mathrm{NaOH}$ solution was mixed with field concentration $(0.01 \%)$ of pesticide, kept under a magnetic stirrer for two hours at ambient condition. After stirring, the content was centrifuged, and the collected pellet was lyophilized, used for further studies.

\subsection{Preparation of cellulose grafted curcumin composite.}

Cellulose grafted curcumin composite was prepared by the method of simple dispersion. Initially, crude curcumin was extracted from the rhizome of Curcuma longa. The rhizome was soaked in ethanol, kept in the shaker at ambient conditions for $24 \mathrm{hrs}$. After the incubation period, the content was filtered through filter paper, and the collected filtrate was concentrated, and the concentrated extract was used for composite preparation. In a typical procedure of composite preparation, a known quantity of extracted cellulose $(100 \mathrm{mg})$ dispersed in $1.0 \mathrm{M} \mathrm{NaOH}$ solution was mixed with $0.1 \mathrm{~g}$ of crude curcumin, thus obtained, the reaction mixture kept under magnetic stirrer for two hours at ambient condition. After stirring, the content was centrifuged, and the collected pellet was lyophilized, used for further studies. 


\subsection{Characterization.}

Characterization of the prepared composite was done by Fourier transform spectroscopy (FTIR), scanning electron microscopy (SEM), and X-ray diffraction (XRD) studies to determine functional groups, morphology (size \&shape), and crystallinity. BrukerOpttic GmbH Tensor 27 was used to record FTIR spectra (400-4000 cm-1.). SEM micrograph was taken with Supra 55-Carl Zeiss (Germany) magnification range of 35-10,000, resolution $200 \mathrm{~A}$, acceleration voltage $19 \mathrm{kV}$. Crystallinity was determined from the XRD Rigaku smart lab instrument, which operated at a voltage of $40 \mathrm{kV} 30 \mathrm{~mA}$ current, $\mathrm{Cu} \mathrm{Ka}^{-1}$ radiations.

\subsection{Qualitative release profile studies.}

The effect of solvents on the release of chlorpyrifos, curcumin was studied under in vitro condition. In this method, the known volume of respective composite (100mg) was suspended in $5 \mathrm{ml}$ of ethanol, water \& saline followed by incubation at ambient condition under shaking condition. At a defined time period, the suspension was centrifuged at 2500rpm for $15 \mathrm{~min}$ followed by UV-Vis spectrophotometric measurement of collected supernatant at 290nm (Cellulose grafted chlorpyrifos), 424nm (Cellulose grafted curcumin).

\subsection{Compatibility of chlorpyrifos on groundnut.}

Supernatant derived from the respective solvent control release group was studied for compatibility using groundnut adopting seedlings emergence test. The supernatant of the respective solvent group derived at the determined time period was filter-sterilized under aseptic condition. Filtrate thus obtained was transferred to the sterile Petri plate, and the healthy seeds of groundnut were allowed to immerse in the filtrate for 1 hour at ambient condition. After the incubation period, the treated seeds were transferred to the sterile Petri plate lined with sterile moisture filter paper. The Control group was treated with chlorpyrifos $(0.01 \%)$ as a positive group, distilled water as the negative group. Three replicates were maintained in each treatment. All the treatment groups were incubated at $28^{\circ} \mathrm{C}$, and daily observation was recorded to determine seedlings emergence.

\subsection{Antioxidative activity of cellulose grafted curcumin.}

The antioxidative activity of control released suspension derived from cellulose grafted curcumin was studied by DPPH free radical scavenging assay. Reaction mixture - a total volume of $1 \mathrm{ml}$ consist of $0.5 \mathrm{ml}$ of respective solvent derived controlled release supernatant, $125 \mu \mathrm{l}$ prepared DPPH $(1 \mathrm{mM})$, and $375 \mu \mathrm{l}$ methanol. After $30 \mathrm{~min}$ incubation at $25^{\circ} \mathrm{C}$, the decrease in absorbance was measured at $\lambda=517 \mathrm{~nm}$.

\section{Results and Discussion}

Lignocellulosic biomass (LB) is an abundant and renewable resource from plants mainly composed of polysaccharides (cellulose and hemicelluloses) and an aromatic polymer (lignin). LB has a high potential as an alternative to fossil resources to produce secondgeneration biofuels and bio-sourced chemicals and materials without compromising global food security [2]. In this study, cellulose was extracted from processed biomass of water 
hyacinth - lignocellulosic major aquatic weed and studied the potential biological activities under in situ condition.

\subsection{Characterization of extracted cellulose.}

Acid-alkali treatment was adopted for the extraction of cellulose from the collected biomass. Due to the structural complexity of the polycarbohydrates, pre-treatment is required to disrupt the structure of lignocellulose materials. Extraction of cellulose from water hyacinth using $\mathrm{NaOH}$ and $\mathrm{HNO}_{3}$ made cellulose soluble due to the formation of sodium salt and separated it from other constituents, while the acid neutralization regenerates the insoluble cellulose with modified structure process of pure cellulose [18]. Powdered biomass treated with $\mathrm{HNO}_{3}$ and $\mathrm{NaOH}$ brought about highly stable cellulose with a final yield of $22.4 \mathrm{~g} / 100 \mathrm{~g}$ of dry weight (Fig 1a). Extracted cellulose was characterized by FTIR, SEM, and XRD. FTIR analysis reveals that the characteristic peaks at 3252,1633 , and $1336 \mathrm{~cm}^{-1}$, which describes the major functional groups of cellulose (Fig 1b). Scanning electron microscopy analysis shows that the smooth fibrous structure of 10-15 $\mu \mathrm{m}$ (Fig 1c). Further characterization was done by XRD studies (Fig 1d). XRD pattern reveals the highly crystalline nature of the extracted cellulose, which was due to the complete removal of hemicellulose and lignin. The XRD diffractogram reveals that diffraction peaks at 2 Theta $33,35 \& 38$. Similar peaks were recorded at $34.7 \& 39$ (Fig 1d). The crystallography peak at 2 Theta 34.7 corresponds to the (102) plane, while 2 Theta 39 corresponds to the (004) planes. However, it is observed that there was a change in the relative intensity of the amorphous peaks and its width, indicating a change in the crystallinity. The diffraction peak for raw water hyacinth was relatively broader and became sharper and narrower after chemical treatments. This indicates that the content of cellulose and the degree of crystallinity was increased by chemical treatments.

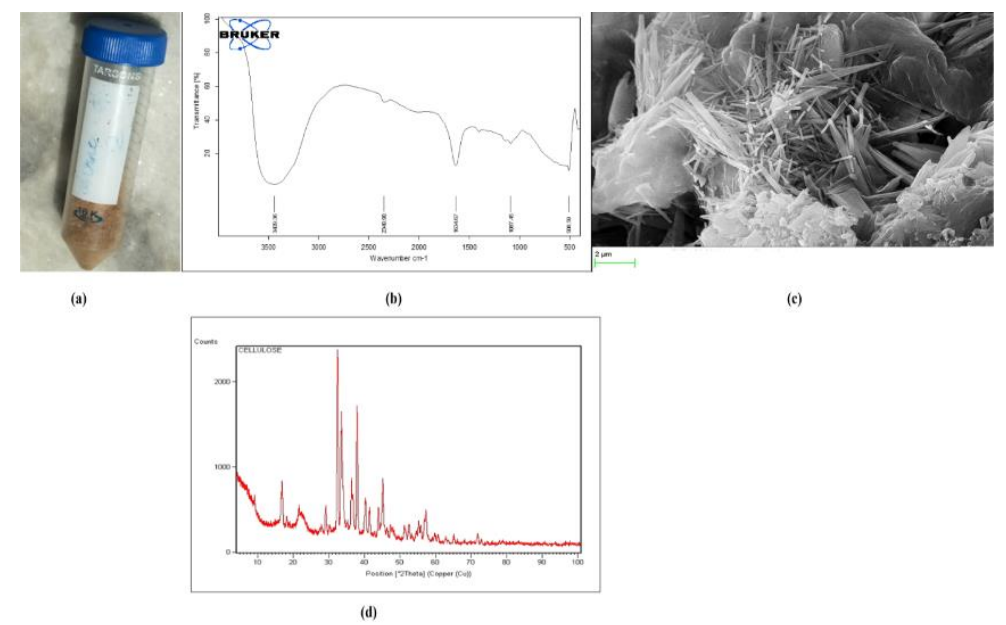

Figure 1.Characterization of cellulose extracted from WHB (a) Extracted cellulose (b) FTIR spectra of cellulose (c) SEM micrograph of extracted cellulose (d) XRD pattern of cellulose.

\subsection{Characterization of cellulose grafted chlorpyrifos and curcumin composites.}

A simple dispersion method was adopted for the preparation of the respective composite, brought about a highly stable structure. The total yield of the cellulose grafted pesticide and curcumin was found to be $2.0 \%$ and $4.25 \% \mathrm{w} / \mathrm{v}$, respectively. Characterization of the respective composite was done by FTIR analysis, which confirms the structural modification. FTIR spectrum of cellulose grafted curcumin shows a characteristic peak at 
$1424.76 \mathrm{~cm}^{-1}$ and $2089.87 \mathrm{~cm}^{-1}$, which are associated with the aromatic group of curcumin (Fig 2a). The observed peaks at $1633 \mathrm{~cm}^{-1}$ and $1638 \mathrm{~cm}^{-1}$ are associated with the presence of a strong alkyl amine group in cellulose grafted pesticide composite (Fig 2b).

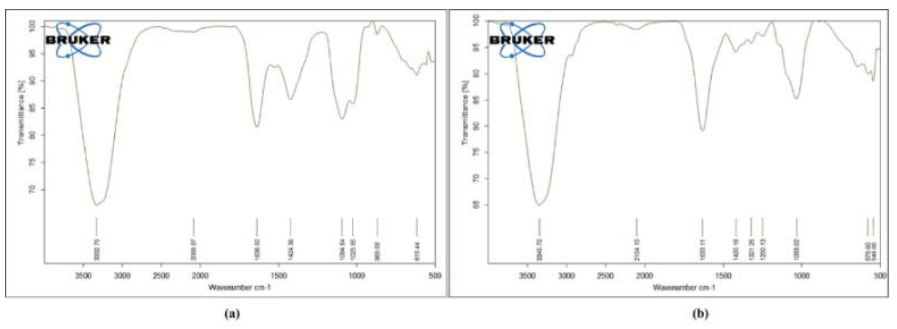

Figure 2. FTIR Spectra of composite (a) cellulose grafted chlorpyrifos (b) cellulose grafted curcumin.

\subsection{Qualitative release profile.}

The effect of solvents on the release of chlorpyrifos and curcumin from the cellulose composite was studied under in vitro condition. Qualitative determination of the release of chlorpyrifos and curcumin was studied by spectrophotometric measurement of the supernatant at $290 \mathrm{~nm}$ and $424 \mathrm{~nm}$, respectively (Table 1). Among the three solvents tested, maximum release at all the tested time periods was recorded in ethanol, followed by PBS. A sharp peak at a particular wavelength was recorded in ethanol treatment. The release was not recorded in distilled water (Fig 3,4).

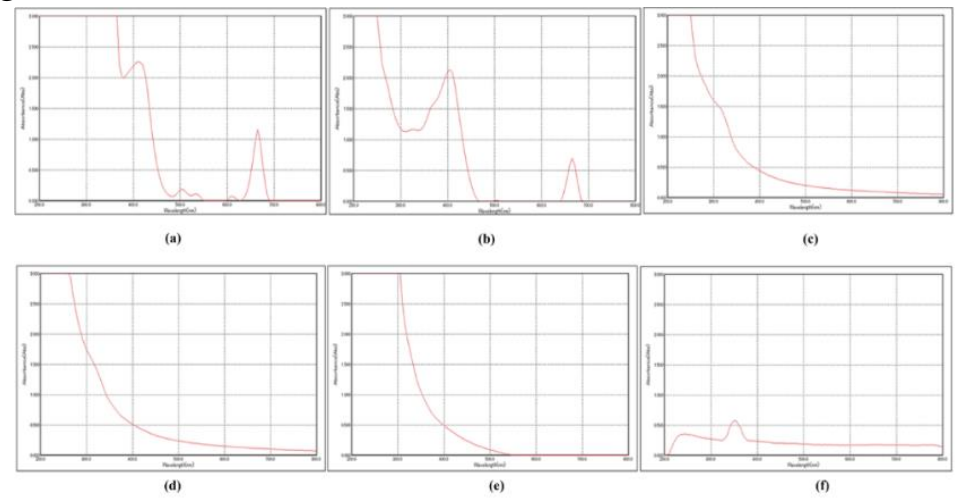

Figure 3. UV visible spectra of curcumin released from the composite in the solvent at the respective time period (a) ethanol-24 hrs (b) ethanol-48 hrs (c) PBS-24 hrs (d) PBS-48 hrs (e) distilled water-24 hrs (f) distilled water-48 hrs.
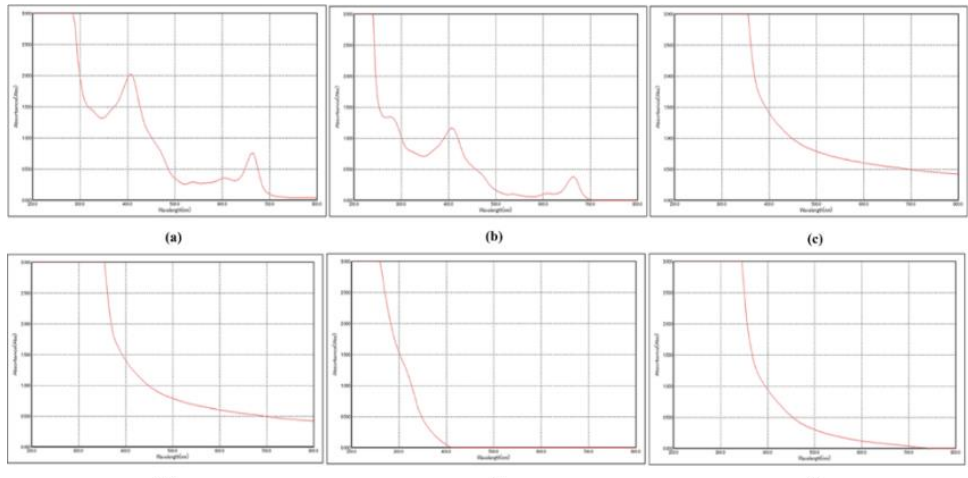

Figure 4. UV visible spectra of chlorpyrifos released from the composite in the solvent at the respective time period (a) ethanol-24 hrs (b) ethanol-48 hrs (c) PBS-24 hrs (d) PBS-48 hrs (e) distilled water-24 hrs (f) distilled water-48 hrs. 
Biological activities of supernatant derived from the respective solvent treatment group were studied. In the case of cellulose grafted chlorpyrifos composite, released suspension derived from the respective solvent was evaluated on the seedling emergence of groundnut. A lower reduction of seedlings emergence was noticed in ethanol released solution followed by PBS. No impact of seedlings emergence was observed in distilled water released solution (Fig $5)$.

The antioxidative activity was studied with control release samples of cellulose grafted curcumin composite. Results indicate that free radicals scavenging activity were found to be maximum in ethanol derived solution at all the tested time periods followed by PBS (Fig 6). These results reveal that the curcumin was effectively released into the dispersion medium (ethanol), followed by PBS, which brought about a notable increase in scavenging activity. Recent studies reveal the potential role of polymers based composite in the biomedicine field as a drug delivery agent and tissue engineering component and other environmental applications due to their unique properties [19-22].

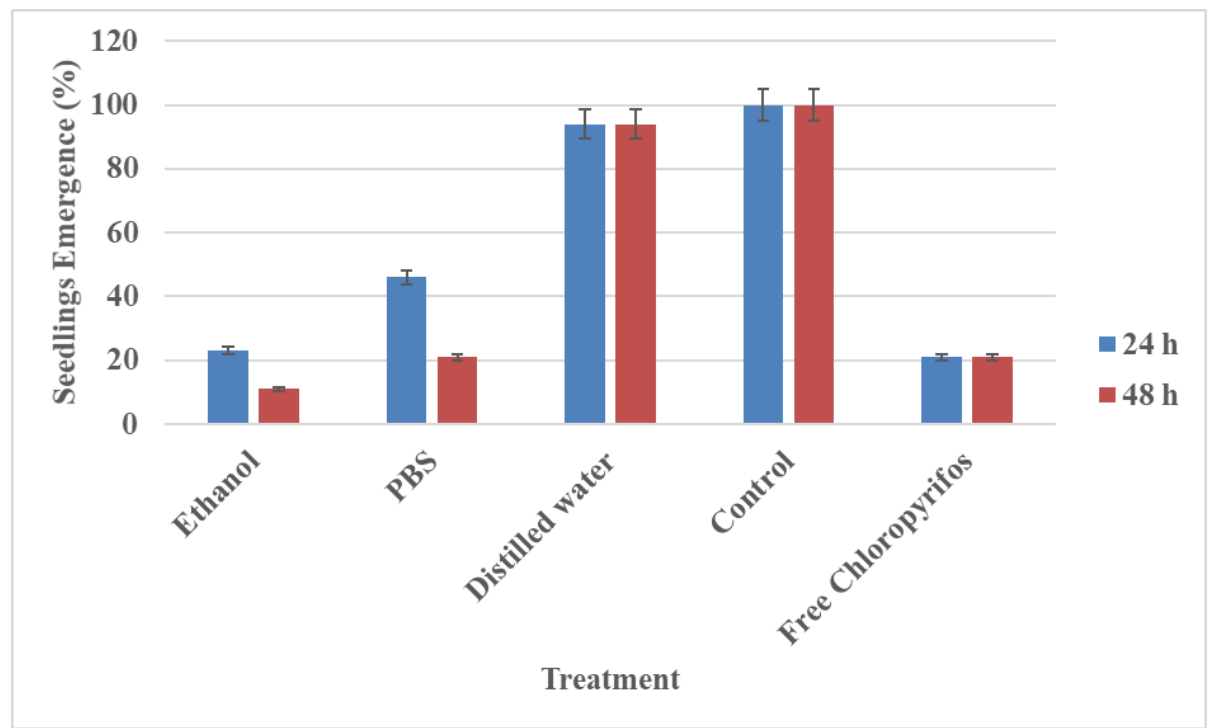

Figure 5. Effect of released pesticides in the solvents at different time periods on the emergence of the seedlings of groundnut.

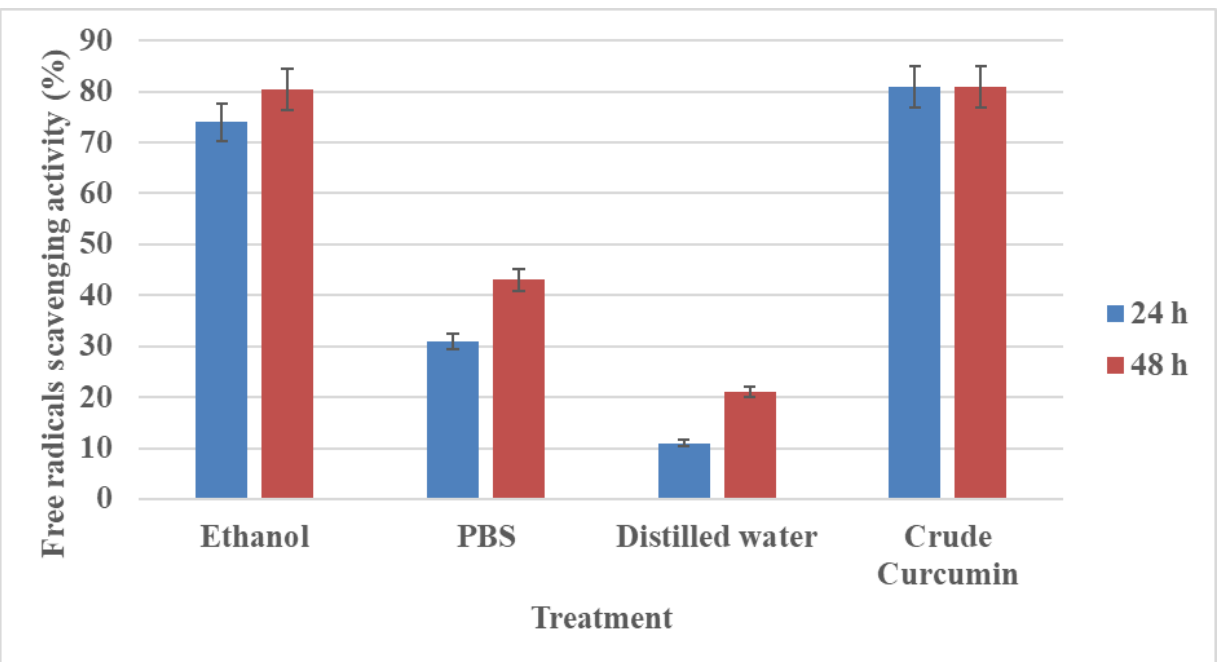

Figure 6. Free radicals scavenging activity of curcumin released in the solvents at different time periods. 
Table 1. Effect of solvents on the release of chlorpyrifos and curcumin from the composite in the solvents at the respective time periods.

\begin{tabular}{l|l|l|l|l}
\hline \multirow{2}{*}{ Solvent tested } & \multicolumn{2}{c|}{ Chlorpyrifos } & \multicolumn{2}{c}{ Curcumin } \\
\cline { 2 - 5 } & $24 \mathrm{hrs}$ & $48 \mathrm{hrs}$ & $24 \mathrm{hrs}$ & $48 \mathrm{hrs}$ \\
\hline Ethanol & +++ & +++ & +++ & +++ \\
\hline Phosphate buffered saline (PBS) & ++ & ++ & ++ & ++ \\
\hline Distilled water & + & + & + & + \\
\hline
\end{tabular}

\section{Conclusions}

The potential biological activities of water hyacinth derived cellulose and its fabricated composite material was studied in this present investigation. Biocompatible polymer cellulose was extracted from water hyacinth biomass adopting acid-alkali treatment, and the extracted cellulose reveals distinct structural, functional properties. Extracted cellulose was fabricated with chlorpyrifos pesticide, crude curcumin and transformed into the stable composite material. The effect of solvents like ethanol,phosphate-buffered saline (PBS), and distilled water on the release of pesticide and curcumin were investigated under laboratory conditions. The release profile of pesticide and curcumin from the fabricated composite material was studied. Suspension derived from ethanol at all the incubation periods reveals the maximum release of pesticide and curcumin. Pesticide released at determined time periods reveals a phytotoxic effect on groundnut, whereas curcumin released exhibited notable free radical scavenging activity. All these findings clearly indicate that the possible utilization of cellulose fabricated composite material for an environmental and medicinal application due to its sustained or controlled release pattern.

\section{Funding}

This research received no external funding.

\section{Acknowledgments}

We acknowledge SIST, Chennai, Tamil Nadu, India, for the composite characterization studies.

\section{Conflicts of Interest}

The authors declare no conflict of interest.

\section{References}

1. Ochaikul, D.; Jongmeesuk, A.; Maneeruttanarungroj, C. Bioethanol Fermentation from Acid/Base-Treated Water Hyacinth Biomass using Fermentation Yeasts Saccharomyces cerevisiae YRK 017 and Candida shehatae ATCC 22984.Int. J. Renew, Energy Res. 2019, 9, 1428-1434. https://dx.doi.org/10.1007/s00449019-02136-3.

2. Kiran Kumar, A; Sharma, S. Recent Updates on Different Methods of Pre-treatment of Lignocellulosic Feedstocks: A Review. Bioresour Bioprocess 2017, 27, 117-129. https://dx.doi.org/10.1186\%2Fs40643017-0137-9.

3. Munive-Olarte, A.; Rosano-Ortega, G.; Schabes-Retchkiman, P.; Martinez-Gallegos, M.S.M.; Kassis, E. El; Gonzalez-Perez, M.; Pacheco-Garcia, F. Assessment of Biomass of Leaves of Water Hyacinth (Eichhornia crassipes) as Reducing Agents for the Synthesis of Nanoparticles of Gold and Silver.Int. J. Adv. Eng. Manag. Sci. 2017, 3, 364-370. https://dx.doi.org/10.24001/ijaems.3.4.14.

4. Hemalatha, S.; Makeswari, M. Green synthesis, characterization and antibacterial studies of $\mathrm{CuO}$ nanoparticles from Eichhornia crassipes. Rasayan J. Chem. 2017, 10, 838-843. http://dx.doi.org/10.7324/RJC.2017.1031800. 
5. Cuervo Blanco, T.; Sierra Ávila, C.A.; ZeaRamírez, H.R. Nanostructured $\mathrm{MnO}_{2}$ catalyst in E. crassipes (water hyacinth) for indigo carmine degradation. Rev. Colomb. Química.45, 30 2016, 45, 30-38. http://dx.doi.org/10.15446/rev.colomb.quim.v45n2.60395.

6. Awasthi, M.; Kaur, J.; Rana, S. Bioethanol Production Through Water Hyacinth, Eichhornia crassipes Via Optimization of the Pre-treatment Conditions. Int. J. Emerging Technology Advanced Engineering 2013, 3, 42-46.

7. Kaur, M.; Kumar, M.; Sachdeva, S. Puri, S.K. Aquatic weeds as the next generation feedstock for sustainable bioenergy production. $\quad$ Bioresour.Technol. 2018, 390-402. http://dx.doi.org/10.1016/j.biortech.2017.11.082.

8. Agarwal, U.P.; Ralph, S.A.; Reiner, R.S.; Baez, C. Probing crystallinity of never-dried wood cellulose with Raman spectroscopy. Cellulose 2016, 23, 125-144. https://doi.org/10.1007/s10570-015-0788-7.

9. Zhang, Y.Y.; Zhang, D.Y.; Barrett, S.C.H. Genetic uniformity characterizes the invasive spread of water hyacinth (Eichhornia crassipes), a clonal aquatic plant. Mol. Ecol. 2010, 19, 1774-1786 http://dx.doi.org/10.1111/j.1365-294X.2010.04609.x.

10. Sotolu, A.O. Management and utilization of weed: Water hyacinth (Eichhornia crassipes) for improved aquatic resourcesJ. Fish. Aquat. Sci. 2012, 8, 1-8. http://dx.doi.org/10.3923/jfas.2013.1.8.

11. Bhattacharya, A.; Sadhukhan, A.K.; Ganguly, A.; Chatterjee, P.K. Dilute Acid Induced Changes on Microscopic and Tomographic Structure of Water Hyacinth [Eichhornia crassipes (Mart.) Solms] Biomass During Bioconversion Process to Xylitol.Indian Journal of Science and Technology. 2016, 9, 1-9. https://doi.org/10.17485/ijst/2016/v9i6/82888.

12. Muralidharan, R.; Radha, K. V. A kinetic study of polyhydroxybutyrate production on nitrogen limited medium using Bacillus subtilis MTCC 9763 through a two stage cultivation strategy. J. Environ. Biol. 2015, 36, 537-542.

13. Sindhu, R.; Binod, P.; Pandey, A.; Madhavan, A.; Alphonsa, J.A.; Vivek, N.; Gnansounou, E.; Castro, E.; Faraco, V. Water hyacinth a potential source for value addition: An overview. Bioresour. Technol. 2017, 230, 152-162. https://doi.org/10.1016/j.biortech.2017.01.035.

14. Vanathi, P.; Rajiv, P.; Sivaraj, R. Synthesis and characterization of Eichhornia-mediated copper oxide nanoparticles and assessing their antifungal activity against plant pathogens.Bull. Mater. Sci. 2016, 39, 1165-1170. http://dx.doi.org/10.1007/s12034-016-1276-x.

15. Preethi, K.; Umesh, M. Water Hyacinth: A Potential Substrate for Bioplastic (PHA) Production Using Pseudomonas aeruginosa. Int. J. Appl. Res. 2015, 1, 349-354.

16. Bhattacharya, S.; Mandal, L.; Mukherjee, B.; Lopamudra, D.; Ehsan, I.; Debnath, M.C.; Raghuvir, H.G.; Murari, M.P.; Majumdar, S. Apigenin loaded nanoparticle delayed development of hepatocellular carcinoma in rats. Nanomedicine, 2018, 14, 1905-1917. http://dx.doi.org/10.1016/j.nano.2018.05.011.

17. Silva, A.; Martínez-Gallegos, S.; Rosano-Ortega, G.; Schabes-Retchkiman, P.; Vega-Lebrún, C.; Albiter, V. Nanotoxicity for $E$. coli and characterization of silver quantum dots produced by biosynthesis with Eichhorniacrassipes. J. Nanostructures. 2017, 7, 1-12. http://dx.doi.org/10.22052/jns.2017.01.001.

18. Ruan, T.; Zeng, R.; Yin, X.-Y.; Zhang, S.-X.; Yang, Z.-H. Water Hyacinth (Eichhornia crassipes) Biomass as a Biofuel Feedstock by Enzymatic Hydrolysis. BioResources, 2016, 11, 2372-2380. https://doi.org/10.15376/biores.11.1.2372-2380.

19. Arul Maximus, R.; Karthick Raja Namasivayam, S.; Prasana, M.; Arvind Bharani, R.S. A green chemistry to produce iron oxide - Chitosan nanocomposite (CS-IONC) for the upgraded bio-restorative and pharmacotherapeutic activities - Supra molecular nanoformulation against drug-resistant pathogens and malignant growth. International Journal of Biological Macromolecules. 2019, 138, 1109-1129, https://doi.org/10.1016/j.ijbiomac.2019.07.158.

20. Nasef, S. M.; Khozemy, E. E.; Mahmoud, G. A. Characterisation and in vitro drug release properties of chitosan/acrylamide/gold nanocomposite prepared by gamma irradiation. International Journal of Polymeric $\begin{array}{lllll}\text { Materials and } \quad \text { Polymeric } & \text { Bomaterials, }\end{array}$ https://doi.org/10.1080/00914037.2018.1493685.

21. Eisa, W. H.; Abdelnaby, T.; Mostafa, S.; Elzayat, M. Y. In situ preparation of chitosan/gold nanocomposite: Structural and catalytic properties. Advances in Polymer Technology, 2018, 37, 2095-2101, https://doi.org/10.1002/adv.21867.

22. Karthick Raja Namasivayam, S.; Gayathri, V.; Arvind Bharani, R.S. 2020. Immuno biocompatibility and anti-quorum sensing activities of chitosan-gum acacia gold nanocomposite (CS-GA-AuNC) against Pseudomonas aeruginosa drug-resistant pathogen. Sustainable Chemistry and Pharmacy 17 2020, 17, 100300, https://doi.org/10.1002/j.scp.2020.100300. 